\title{
Picroside II prevents inflammation injury in mice with diabetic nephropathy via TLR4/NF-אB pathway
}

\author{
Chunmei $\mathrm{Ma}^{1}$ and Aijie Shi ${ }^{2 *}$ \\ ${ }^{1}$ Department of Integrated traditional Chinese and Western Medicine, Jinan Central Hospital, Jinan City, Shandong \\ Province, China; ${ }^{2}$ Department of Renal Medicine, Tianjin Dongli Hospital, Tianjin, China
}

"Corresponding author: Aijie Shi, Department of Renal Medicine, Tianjin Dongli Hospital, No. 245 Jintang Road and East of the Outer Ring Crossing, Tianjin 300300, China. Email: ajshi9485@163.com

Received: 29 September 2021; Accepted: 26 October 2021; Published: 27 November 2021 (c) 2021 Codon Publications

OPEN ACCESS @C) ORIGINAL ARTICLE

\begin{abstract}
The purpose of this study was to investigate the therapeutic effects of picroside II on diabetic nephropathy and reveal the involved underlying signal pathway. Male Sprague-Dawley (SD) mice were used to construct an animal model of streptozotocin (STZ)-induced diabetic nephropathy. Body weight and fasting blood glucose values were recorded. Enzyme-linked immunosorbent assay (ELISA) was used to measure the levels of proteinuria, blood urea nitrogen (BUN), serum creatinine (Scr), interleukin (IL)-1 $\beta$, IL-6, monocyte chemoattractant protein-1 (MCP1 ) and necrosis factor alpha (TNF- $\alpha$ ). Protein expression was determined using Western blotting test. Hematoxylin and eosin (H\&E) staining was used to examine the morphological changes in kidney tissues. Treatment with picroside II (10 and $20 \mathrm{mg} / \mathrm{kg}$ ) increased the STZ-induced reduction in body weight of diabetic mice. It also reversed the elevation of fasting blood glucose in STZ-induced diabetic mice. The levels of proteinuria, BUN and Scr were significantly increased in STZ-induced diabetic mice and these increments were prevented by picroside II. The serum levels of MCP-1, IL-1 $\beta$, IL- 6 and TNF- $\alpha$ were reduced, and the morphological damage was lessened by Picroside II in mice with diabetic nephropathy. Besides, picroside II prevented the activation of TLR4/NF-kB pathway. This study proved that picroside II inhibited inflammatory response and prevented kidney injury in mice with diabetic nephropathy through modulation of TLR4/NF-kB pathway, indicating beneficial effect of picroside II on diabetic nephropathy.
\end{abstract}

Keywords: picroside II; inflammation injury; diabetic nephropathy; TLR4/NF-kB pathway

\section{Introduction}

Diabetic nephropathy is one of the complications caused by diabetes mellitus, primarily contributing to the endstage renal failure worldwide (Umanath and Lewis, 2018). Around $30-40 \%$ of diabetic patients develop diabetic nephropathy, and its incidence and related deaths have increased rapidly in the past decades (Umanath and Lewis, 2018; Xiong and Zhou, 2019). Hypertension and hyperglycemia are two major risk factors of diabetic nephropathy (John, 2016). Therefore, treatment strategies of diabetic nephropathy include the following: reduction of cardiovascular risk, decrease of blood glucose and blood pressure, and inactivation of reninangiotensin-aldosterone system (RAAS) (Umanath and
Lewis, 2018). Accumulating evidence has revealed that the pathogenesis of diabetic nephropathy is associated with the activation of inflammatory pathways (Wada and Makino, 2013). Thus, anti-inflammatory therapy is also a potential treatment strategy for diabetic nephropathy.

Toll-like receptor 4 (TLR4)/nuclear factor kappa-lightchain enhancer of activated B cells (NF-kB) pathway is an essential inflammatory pathway activated by elevation of reactive oxygen species (ROS), which are produced by activation of RAAS (Xiong and Zhou, 2019). After stimulation, TLR4 transduces the signal and activates the downstream NF- $\mathrm{kB}$ signal pathway (Ni et al., 2020). As a transcription factor, activation of NF- $\mathrm{kB}$ regulated inflammatory gene expression, including increasing pro-inflammatory 
and reducing anti-inflammatory cytokines, resulting in the enhancement of inflammatory response (Zusso et al., 2019). TLR4/NF-kB pathway has been proved to play a key role in diabetic nephropathy-related inflammation (Kintu et al., 2019; Yang et al., 2017). Therefore, inactivation of TLR4/NF-KB pathway is important to prevent the progression of diabetic nephropathy.

Picroside II is a bioactive flavonoid extracted from Pseudolysimachion rotundum var. subintegrum (Plantaginaceae), a traditional Chinese herb with anti-inflammatory properties (Lee et al., 2019). Picroside II has demonstrated its anti-inflammatory effects through multiple signaling pathways in various inflammatory disorders. For example, Picroside II ameliorated airway inflammation through GATA/Th2 pathway in house dust mites (HDM)-induced allergic asthma (Choi et al., 2016). Picroside II prevented the kidney by the repression of TLR4/NF- $\mathrm{KB}$ pathway in oxidative stressinduced renal ischemia and reperfusion injury (Wang et al., 2015). However, no study has explored the role of picroside II in regulating TLR4/NF- $\mathrm{KB}$ pathway during the progression of diabetic nephropathy. Therefore, the purpose of this study was to investigate the therapeutic effects of picroside II in diabetic nephropathy and reveal the involved underlying signal pathway, aiming to provide a new complimentary treatment option for diabetic nephropathy.

\section{Methods}

\section{Animal model}

Male Sprague-Dawley (SD) mice (180-220 g, $\mathrm{n}=24$ ) were provided by Shandong Experimental Animal Center (Jinan city, Shandong Province, China). The mice were raised at $23^{\circ} \mathrm{C} \pm 2{ }^{\circ} \mathrm{C}$ in a humidity of $40 \% \pm 5 \%$ under 12 -h lightdark cycle for 1 week to accommodate new environment. Ethical approval was obtained from the Ethics Committee of Qilu Hospital of Shandong University. All animal procedures were conducted in compliance with the ethical standards under the protocol approved by the Ethics Committee of Qilu Hospital of Shandong University, and were executed according to the Guide for the Care and Use of Laboratory Animals, 8th edition (National Research Council of the National Academies, 2011).

Mice were randomly assigned into four groups $(\mathrm{n}=6$ mice/group). Streptozotocin (STZ, $60 \mathrm{mg} / \mathrm{kg}$; AmyJet, Wuhan, Hubei Province, China) was injected intravenously to mice to induce type 2 diabetes. After 3 days, mice were injected with 10 and $20 \mathrm{mg} / \mathrm{kg}$ of picroside II (Leyan Technology, Chengdu, Sichuan Province, China) by intragastric administration once per day for 8 weeks. The body weight and fasting blood glucose were measured at 0, 2, 4, 6 and 8 weeks. After 8 weeks, mice were euthanized by an overdose of chloral hydrate (400 $\mathrm{mg} / \mathrm{kg}$; Sigma-Aldrich, USA). The serum and kidney tissues were collected for additional experiments. The rat urine samples were also collected after 8 weeks for urinary protein measurement.

\section{Hematoxylin and Eosin (H\&E) Staining}

The fresh kidney tissues were fixed using 4\% paraformaldehyde fix solution (Beyotime, Shanghai, China) at $4^{\circ} \mathrm{C}$ for 2 days. The fixed tissues were embedded in paraffin wax. The wax was then cut into $5-\mu \mathrm{m}$ sections and dried on slides. Xylene was used to deparaffinize the wax section, and graded ethanol was used to dehydrate samples. The slides were stained using H\&E staining kit (Beyotime) according to manufacturer's protocol.

\section{Enzyme-linked immunosorbent assay (ELISA)}

ELISA was used to measure proteinuria, blood urea nitrogen (BUN), serum creatinine (Scr), interleukin (IL)-1 $\beta$, IL-6, monocyte chemoattractant protein-1 (MCP-1) and necrosis factor alpha (TNF- $\alpha$ ) using commercial kits (R\&D Systems, Minneapolis, MN, USA) following manufacturer's instructions.

\section{Western blotting test}

Total proteins were extracted from the kidney tissues using ProteoPrep total extraction sample kit (Merck KGaA, Darmstadt, Germany). BCA protein assay kit (Merck KGaA) was used to measure protein concentrations. Proteins were separated using Criterion XT precast gels (Bio-Rad, Hercules, CA, USA) by electrophoresis. Proteins were transferred from gels to polyvinylidene difluoride (PVDF) membranes (Bio-Rad). The membranes were blocked using $5 \%$ bovine serum albumin (BSA) blocking buffer (Solarbio Science \& Technology Co. Ltd., Beijing, China). The membranes were then incubated with primary antibodies, followed by probing using secondary antibodies. Primary antibodies (Abcam, Cambridge, UK) used in this study were: TLR4 (ab13867, 1:500 dilution), p-p65 (ab76302, 1:1000 dilution), p65 (ab16502, 1:800 dilution), $\mathrm{p}$-ІкB $\alpha$ (ab92700, 1:1500 dilution), ІкB $\alpha$ (ab32518, 1:1000 dilution) and GAPDH (Glyceraldehyde 3-phosphate dehydrogenase; ab125247, 1:5000 dilution). The protein signals were tested using ECL luminescence reagent (Absin, Shanghai, China) by iBright Imaging Systems (Thermo Fisher Scientific, Madison, WI, USA).

\section{Statistical analysis}

All results were analyzed using the GraphPad Prism 7.0 software (GraphPad Software, La Jolla, CA, USA). Student's $t$-test and one-way ANOVA were used to 
perform statistical differences between two groups and multiple groups, respectively. All data were presented as mean \pm standard deviation. For measurements, $P<0.05$ was considered statistically significant.

\section{Results}

\section{Treatment with picroside II reduced fasting blood glucose levels in mice with diabetic nephropathy}

The body weight was significantly reduced in STZinduced diabetic mice (Figure 1A). Treatment with picroside II (10 and $20 \mathrm{mg} / \mathrm{kg}$ ) prevented reduction of bodyweight in STZ-induced diabetic mice (Figure 1A). The fasting blood glucose was elevated in STZ-induced diabetic mice compared to that in the control group (Figure 1B). Treatment with picroside II (10 and $20 \mathrm{mg} /$ $\mathrm{kg}$ ) inhibited the elevation of fasting blood glucose in STZ-induced diabetic mice (Figure 1B). Therefore, picroside II reduced STZ-induced elevation of fasting blood glucose in mice with diabetic nephropathy.

\section{Treatment with picroside II lessened kidney injury in mice with diabetic nephropathy.}

The levels of proteinuria, BUN and Scr were significantly increased in STZ-induced diabetic mice (Figures $2 \mathrm{~A}-2 \mathrm{C}$ ). However, treatment with picroside II (10 and $20 \mathrm{mg} / \mathrm{kg}$ ) reduced the levels of proteinuria, BUN and Scr in STZ-induced diabetic mice (Figures 2A-2C). Treatment with picroside II also lessened morphology damage in mice with diabetic nephropathy (Figure 2D). In STZ-induced diabetic mice, the kidney tissues were seriously damaged due to thickening of glomerular basement membrane (GBM), tubular vacuolization and necrosis, widened renal interstitium and inflammatory cell infiltration (Figure 2D). In diabetic mice treated with picroside II, the glomerular volume was normal, the epithelium of renal tubules was smooth and intact, the arrangement of epithelial cells was regular and the inflammatory cell infiltration was reduced (Figure 2D). Therefore, picroside II lessened kidney injury in mice with diabetic nephropathy.

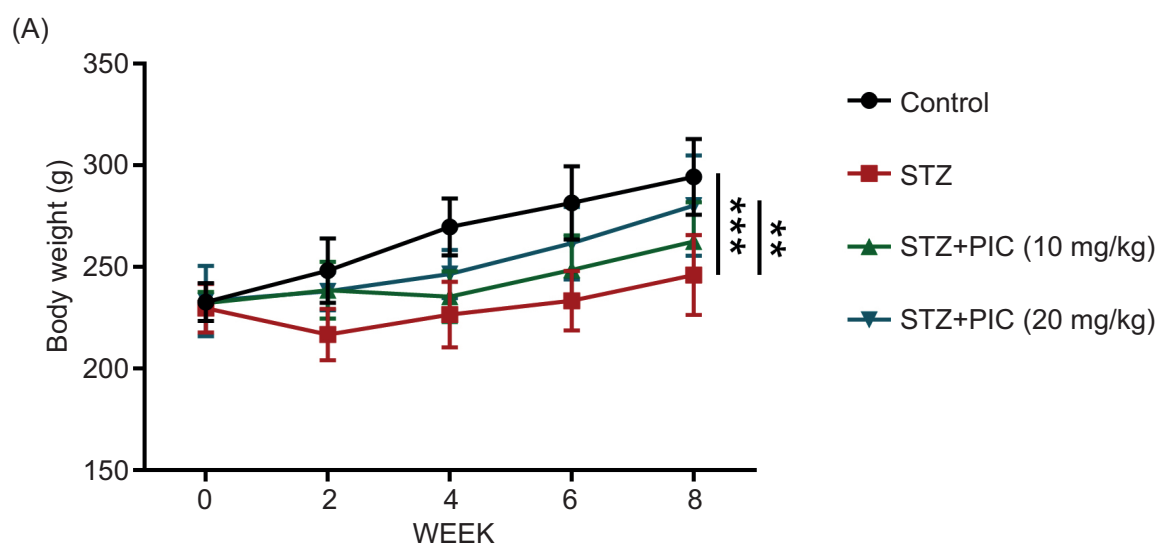

(B)

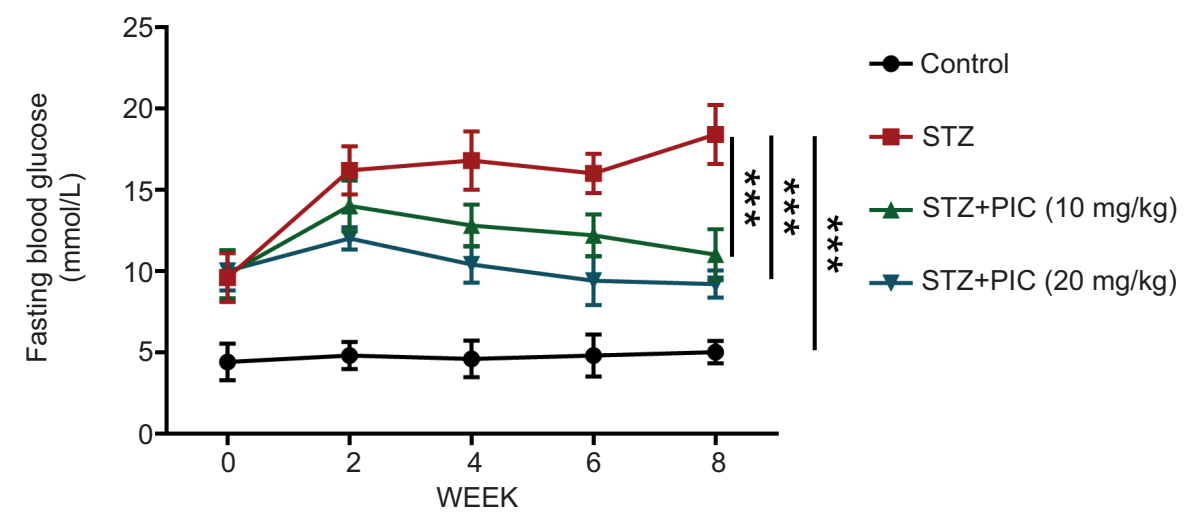

Figure 1. Picroside II treatment reduced fasting blood glucose level in mice with diabetic nephropathy. (A) Picroside II prevented reduction of body weight in mice with diabetic nephropathy. (B) Picroside II inhibited the elevation of fasting blood glucose in mice with diabetic nephropathy. STZ: streptozotocin; PIC: picroside II. ${ }^{* *} P<0.01 ;{ }^{* *} P<0.005$. 
(A)

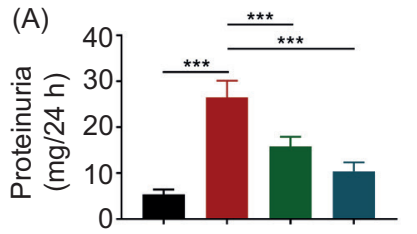

(B)
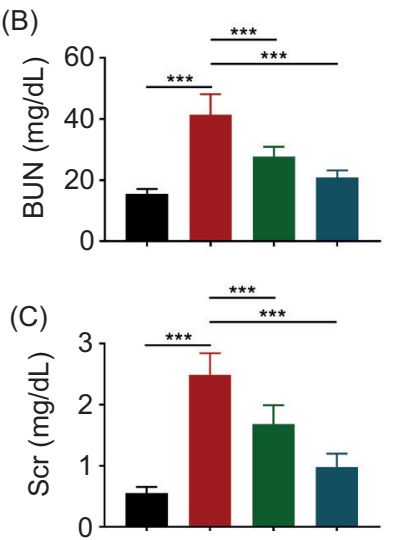

- Control

STZ

STZ + PIC (10 mg/kg)

- STZ + PIC (20 mg/kg)

\section{- Control}

- STZ

a STZ + PIC (10 mg/kg)

- STZ + PIC (20 mg/kg)

- Control

- STZ

- STZ + PIC (10 mg/kg)

n STZ + PIC (20 mg/kg)
(D)

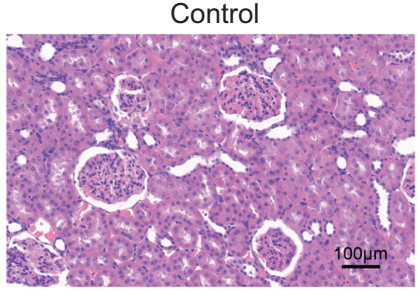

$\mathrm{STZ}+\mathrm{PIC}(10 \mathrm{mg} / \mathrm{kg})$

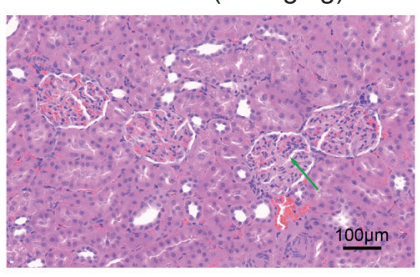

STZ

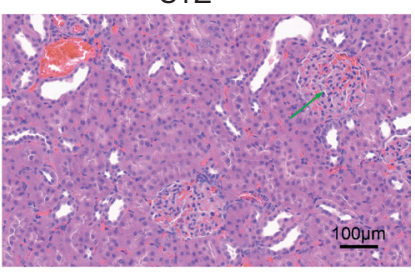

$\mathrm{STZ}+\mathrm{PIC}(20 \mathrm{mg} / \mathrm{kg})$

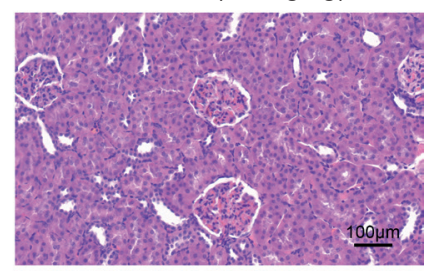

Figure 2. Picroside II treatment lessened kidney injury in mice with diabetic nephropathy. (A) Picroside II inhibited the elevation of proteinuria in mice with diabetic nephropathy. (B) Picroside II inhibited the elevation of BUN in mice with diabetic nephropathy. (C) Picroside II inhibited the elevation of Scr in mice with diabetic nephropathy. (D) Picroside II lessened morphological changes in mice with diabetic nephropathy. ${ }^{* * *} P<0.005$.
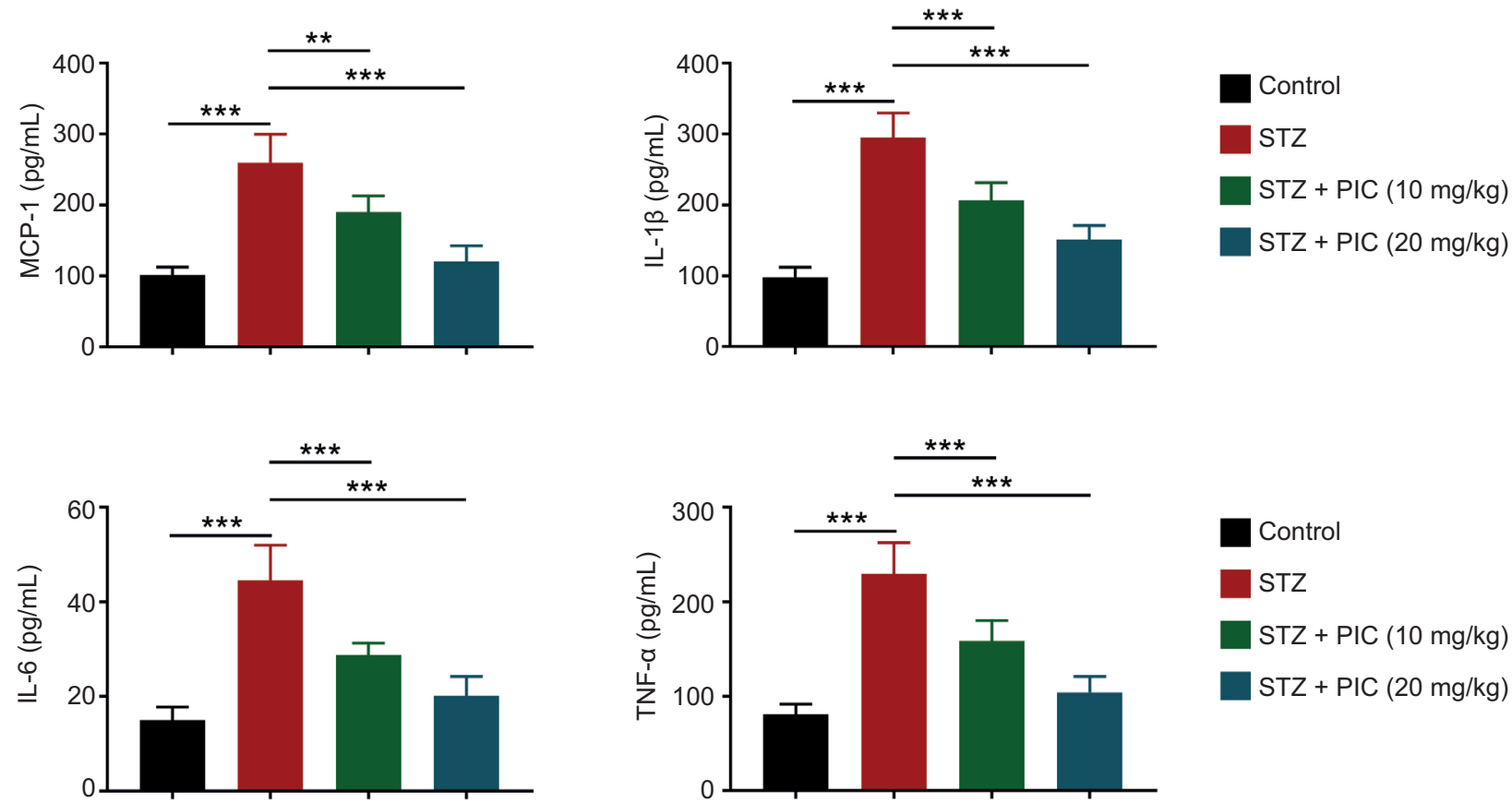

Control

STZ

STZ + PIC (10 mg/kg)

$\mathrm{STZ}+\mathrm{PIC}(20 \mathrm{mg} / \mathrm{kg})$

Figure 3. Picroside II treatment suppressed inflammatory response in mice with diabetic nephropathy. Picroside II inhibited the elevation of MCP-1, IL-1 $\beta$, IL- 6 and TNF- $\alpha$ in mice with diabetic nephropathy. ${ }^{* *} P<0.01 ;{ }^{* * *} P<0.005$.

Treatment with picroside II suppressed inflammatory response through modulation of TLR4/NF- $\otimes B$ pathway in mice with diabetic nephropathy

In mice with diabetic nephropathy, the serum levels of MCP-1, IL-1 $\beta$, IL- 6 and TNF- $\alpha$ were significantly increased (Figure 3). However, treatment with picroside II reduced the serum levels of MCP-1, IL-1 $\beta$, IL- 6 and TNF- $\alpha$ in mice with diabetic nephropathy (Figure 3). Upregulation of TLR4, p-p65 and p-IкB $\alpha$ and downregulation of ІкB $\alpha$ were observed in mice with diabetic nephropathy (Figure 4). Treatment with picroside II 


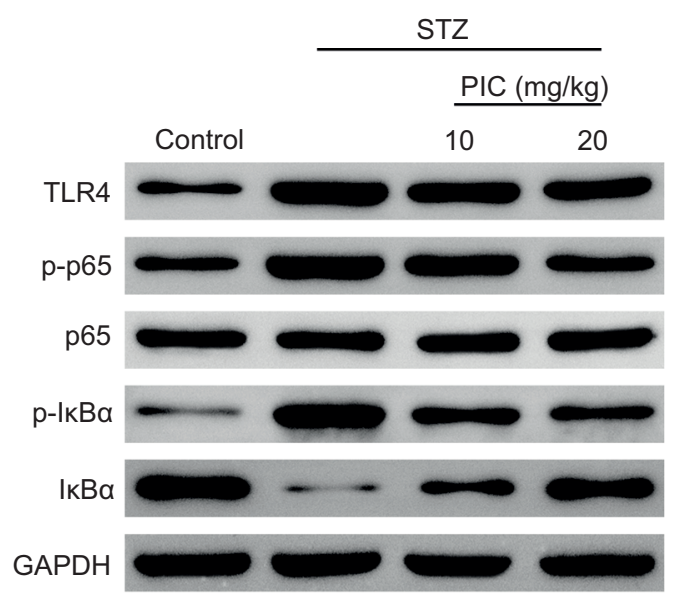

Control

STZ

$\mathrm{STZ}+\mathrm{PIC}(10 \mathrm{mg} / \mathrm{kg})$

$\mathrm{STZ}+\mathrm{PIC}(20 \mathrm{mg} / \mathrm{kg})$
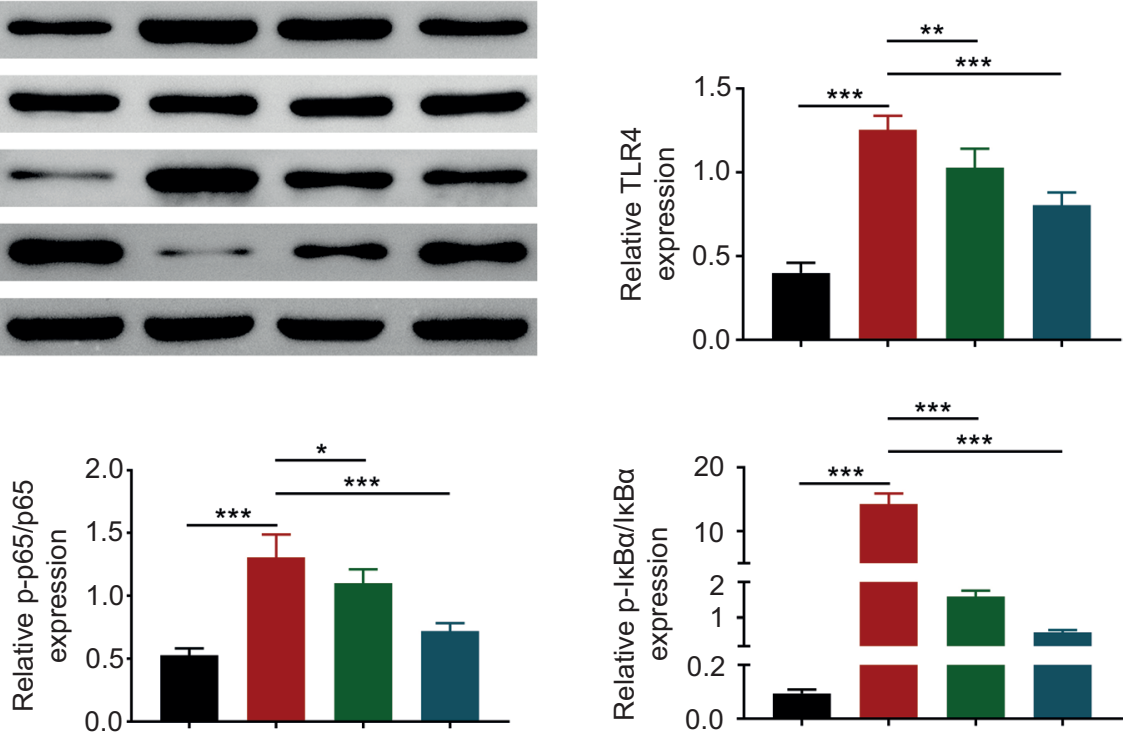

Figure 4. Picroside II treatment modulated TLR4/NF-кB pathway in mice with diabetic nephropathy. Picroside II attenuated the upregulation of TLR4, $p$-p65 and $p$ - $\mid \kappa B \alpha$ and down-regulation of $1 \kappa B \alpha$ in mice with diabetic nephropathy. ${ }^{*} P<0.05 ;{ }^{* *} P<0.01 ;{ }^{* *} P<0.005$.

prevented alterations in the expressions of these proteins (Figure 4). The data demonstrated that picroside II suppressed inflammatory response through modulation of TLR4/NF-KB pathway in mice with diabetic nephropathy.

\section{Discussion}

The activation of inflammatory reaction is a major factor contributing to the progression of diabetic nephropathy (Wada and Makino, 2013). Therefore, inhibition of inflammatory response is an effective solution for the prevention and treatment of diabetic nephropathy. In this study, picroside II was found to prevent reduction in body weight and reversed the elevation of fasting blood glucose level in STZ-induced diabetic mice. Picroside II inhibited morphological damage and parameters of kidney injury, and reduced the production of pro-inflammatory cytokines. Additional experiments also found that picroside II suppressed the activation of TLR4/NF-kB pathway. Thus, data of this study indicated that picroside II suppressed inflammatory response and kidney injury through blocking the signal transduction of TLR4/NF-kB pathway. The data suggested that picroside II could be a potential complementary therapy for the prevention and treatment of diabetic nephropathy.

Streptozotocin is an antibiotic that can induce pancreatic $\beta$-cell damage and is widely used to construct diabetic animal model (Kitada et al., 2016). It is a useful model to investigate early alterations in diabetic nephropathy (Kitada et al., 2016). Thus, STZ was used to induce diabetic nephropathy in SD mice. Loss in body weight and elevation in fasting blood glucose were observed in STZ-treated mice, implying the successful construction of diabetic rat model. Treatment with picroside II prevented both loss in body weight and elevation in fasting blood glucose level in STZ-induced diabetic mice, implying the protective effects of picroside II against diabetic nephropathy.

Morphological changes in diabetic nephropathy included thickening of GBM, tubulointerstitial fibrosis, tubular vacuolization and necrosis, and interstitial inflammation (Qi et al., 2017). These changes were also observed in the present study in mice with diabetic nephropathy. Treatment with picroside II lessened these histological changes in kidney tissues in a dose-dependent manner, indicating that picroside II ameliorated kidney injury during diabetic nephropathy. Increase in proteinuria, BUN and Scr are prime indicators of kidney injury during diabetic nephropathy (Tervaert et al., 2010). Administration of picroside II was found to reduce the levels of proteinuria, BUN and Scr in rat with diabetic nephropathy, further confirming that picroside II ameliorated kidney injury during diabetic nephropathy.

$\mathrm{NF}-\mathrm{kB}$ (p65) is a transcription factor that regulates gene expressions. After stimulation, p65 was phosphorylated 
and IкB kinase was activated, followed by the phosphorylation of ІкB $\alpha$ to induce proteasomal degradation of Ік $\mathrm{B} \alpha$, leading to the transcription of pro-inflammation cytokines and cell adhesion molecules (Cai et al., 2021). In this study, the TLR4/NF-KB pathway was activated due to diabetic nephropathy. Treatment with picroside II suppressed TLR4 expression and phosphorylation of p65

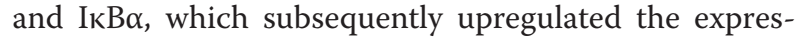
sion of IкB $\alpha$, indicating that picroside II prevented the signal transduction of TLR4/NF-kB pathway, which was consistent with previous findings (Wang et al., 2015). Production of cell adhesion molecule (MCP-1) and pro-inflammation cytokines (IL-1 $\beta$, IL-6 and TNF- $\alpha$ ) was also inhibited by picroside II in a dose-dependent manner. Therefore, the anti-inflammatory effect of picroside II was mediated by repression of TLR4/NF-kB pathway in mice with diabetic nephropathy.

In conclusion, picroside II demonstrated its protective effects in STZ-induced diabetic mice by reducing kidney injury indicators and pro-inflammatory cytokines in a dose-dependent manner. Picroside II also lessened morphological damage of kidney tissues in STZ-induced diabetic mice. The anti-inflammatory effect of picroside II was mediated by repression of TLR4/NF-KB pathway, suggesting that picroside II could be a potential anti-inflammatory drug for preventing and treating diabetic nephropathy.

\section{Competing interests}

The authors state that there are no conflicts of interest to disclose.

\section{Contribution of authors}

Chunmei Ma and Aijie Shi designed the study, and supervised, analyzed and interpreted the data. Both authors prepared, reviewed and approved the final draft of manuscript for publication.

\section{References}

Cai, Y., Zhang, Y., Chen, H., Sun, X.H., Zhang, P., Zhang, L., et al. 2021. MicroRNA-17-3p suppresses NF-kB-mediated endothelial inflammation by targeting NIK and IKK $\beta$ binding protein. Acta Pharmacologica Sinica 2021 Feb 23 https://doi.org/10.1038/ s4:14.01-021-00611-w

Choi, J., Choi, B.K., Kim, J.S., Lee, J.W., Park, H.A., Ryu, H.W., et al. 2016. Picroside II attenuates airway inflammation by downregulating the transcription factor GATA3 and Th2-related cytokines in a mouse model of HDM-induced allergic asthma. PLoS One 11: e0167098. https://doi.org/10.1371/journal.pone.0167098

John, S., 2016. Complication in diabetic nephropathy. Diabetes and Metabolic Syndrome 10: 247-249. https://doi.org/10.1016/j. dsx.2016.06.005
Kintu, A., Abdulla, S., Lubikire, A., Nabukenya, M.T., Igaga, E., Bulamba, F., et al. 2019. Postoperative pain after cesarean section: assessment and management in a tertiary hospital in a low-income country. BMC Health Serv Res. 19(1): 68. https:// doi.org/10.1186/s12913-019-3911-x

Kitada, M., Ogura, Y. and Koya, D., 2016. Rodent models of diabetic nephropathy: their utility and limitations. International Journal of Nephrology and Renovascular Disease 9: 279-290. https:// doi.org/10.2147/ijnrd.S103784.

Lee, K., Choi, J., Choi, B.K., Gu, Y.M., Ryu, H.W., Oh, S.R., et al. 2019. Picroside II isolated from pseudolysimachion rotundum var. subintegrum inhibits glucocorticoid refractory serum amyloid A (SAA) expression and SAA-induced IL-33 secretion. Molecules 24. https://doi.org/10.3390/molecules24102020

National Research Council of the National Academies, 2011. Committee for the update of the guide for the care and use of laboratory animals. In: Guide for the care and use of laboratory animals, 8th edition. The National Academies collection: reports funded by National Institutes of Health. The National Academies Press, Washington, DC.

Ni, W.W., Zhang, Q.M., Zhang, X., Li, Y., Yu, S.s., Wu, H.y., et al. 2020. Modulation effect of Lactobacillus acidophilus KLDS 1.0738 on gut microbiota and TLR4 expression in $\beta$-lactoglobulin-induced allergic mice model. Allergologia et Immunopathologia 48: 149157. https://doi.org/https://doi.org/10.1016/j.aller.2019.06.002

Qi, C., Mao, X., Zhang, Z. and Wu, H., 2017. Classification and differential diagnosis of diabetic nephropathy. Journal of Diabetes Research 2017: 8637138. https://doi.org/10.1155/2017/8637138

Tervaert, T.W., Mooyaart, A.L., Amann, K., Cohen, A.H., Cook, H.T., Drachenberg, C.B., et al. 2010. Pathologic classification of diabetic nephropathy. Journal of the American Society of Nephrology (JASN) 21: 556-563. https://doi.org/10.1681/asn.2010010010

Umanath, K. and Lewis, J.B., 2018. Update on diabetic nephropathy: core curriculum 2018. American Journal of Kidney Diseases (AJKD) 71: 884-895. https://doi.org/10.1053/j.ajkd.2017.10.026

Wada, J. and Makino, H., 2013. Inflammation and the pathogenesis of diabetic nephropathy. Clinical Science (London) 124: 139152. https://doi.org/10.1042/cs20120198

Wang, L., Liu, X.H., Chen, H., Chen, Z.Y., Weng, X.D., Qiu, T., et al. 2015. Picroside II protects rat kidney against ischemia/ reperfusion-induced oxidative stress and inflammation by the TLR4/NF-kB pathway. Experimental and Therapeutic Medicine 9: 1253-1258. https://doi.org/10.3892/etm.2015.2225

Xiong, Y. and Zhou, L., 2019. The signaling of cellular senescence in diabetic nephropathy. Oxidative Medicine and Cellular Longevity 2019: 7495629. https://doi.org/10.1155/2019/7495629

Yang, S., Zhang, J., Wang, S., Zhao, X. and Shi, J., 2017. SOCS2 overexpression alleviates diabetic nephropathy in rats by inhibiting the TLR4/NF-kB pathway. Oncotarget 8: 91185-91198. https:// doi.org/10.18632/oncotarget.20434

Zusso, M., Lunardi, V., Franceschini, D., Pagetta, A., Lo, R., Stifani, S., et al. 2019. Ciprofloxacin and levofloxacin attenuate microglia inflammatory response via TLR4/NF-kB pathway. Journal of Neuroinflammation 16: 148. https://doi.org/10.1186/ s12974-019-1538-9 